3. Acknowledgments. The author gratefully acknowledges the suggestions of Mr. Charles R. Newman for programming the computer.

Naval Ordnance Laboratory

White Oak, Maryland

1. British Association for the Advancement of Science, Mathematical Tables, $v$. 6 : Bessel Functions, Part I, Cambridge University Press, Cambridge, 1950.

2. Harvard Computation Laboratory, Tables of the Bessel Functions of the First Kind of Orders Zero and One, Harvard University Press, Cambridge, Massachusetts, 1947.

3. Ibid., p. xix-xxii.

4. W. G. Bickley \& J. C. P. Miller, Notes on the Evaluation of Zeros and Turning Values of Bessel Functions, Phil. Mag. 36, 1945, p. 121-133.

\title{
Polylogarithms, Dirichlet Series, and Certain Constants
}

\section{By Daniel Shanks}

The polylogarithms $F_{s}(z)$ are defined by

$$
F_{s}(z)=\sum_{m=1}^{\infty} \frac{z^{m}}{m^{s}}
$$

for $|z|<1$ and for the real part of $s \geqq 0$, and by analytic continuation for other values of $z$ and $s$. They can be regarded as functions of $z$, with a parameter $s$, given by the power series (1), or as functions of $s$, with a parameter $z$, given by the Dirichlet series (1).

Recently [1] we discussed the Dirichlet series defined by

$$
L_{a}(s)=\sum_{k=0}^{\infty} \frac{\left(\frac{-a}{2 k+1}\right)}{(2 k+1)^{s}}
$$

and its analytic continuation, where $\left(\frac{-a}{2 k+1}\right)$ is the Jacobi symbol. It is expressible in closed form for three-quarters of all combinations of integers $a$ and $s$; namely, for $s \leqq 1$ and all $a$, for $s$ even and $>1$ if $a<0$, and for $s$ odd and $>1$ if $a>0$.

The remaining, non-closed form $L_{a}(n)$ for $a= \pm 2, \pm 3$, and \pm 6 , with $n \leqq 10$, were computed [1] by a device, which (in essence) is based on the fact that all of the so-called characters modulo 8, 12, or 24 are real. In contrast, the corresponding $L_{a}(n)$ for $a= \pm 5, \pm 7$, and \pm 10 , say, which were also desired, are not obtainable by that method, unless it is modified, since now some of the characters are complex.

We did, however, express $L_{a}(s)$ as a linear combination of the functions $S_{s}(x)$ or $C_{s}(x)$ for various values of $x$ determined by the integer $a$ [1, equations (24)-(27)]. These functions [1, equation (18)] are defined by

$$
\begin{aligned}
& S_{s}(x)=\sum_{k=0}^{\infty} \frac{\sin 2 \pi(2 k+1) x}{(2 k+1)^{s}}, \\
& C_{s}(x)=\sum_{k=0}^{\infty} \frac{\cos 2 \pi(2 k+1) x}{(2 k+1)^{s}} .
\end{aligned}
$$

Received July 23, 1963. 
Now consider the real and imaginary parts of $F_{s}\left(e^{i \pi \alpha / 2}\right)$. We will call them

$$
\begin{aligned}
R_{s}(\alpha) & =\Re F_{s}\left(e^{i \pi \alpha / 2}\right) \\
I_{s}(\alpha) & =g F_{s}\left(e^{i \pi \alpha / 2}\right) .
\end{aligned}
$$

It follows that

$$
\begin{aligned}
& C_{s}(x)=R_{s}(4 x)-\frac{1}{2^{s}} R_{s}(8 x) \\
& S_{s}(x)=I_{s}(4 x)-\frac{1}{2^{s}} I_{s}(8 x) .
\end{aligned}
$$

By the aforementioned linear combinations we may, therefore, express $L_{a}(s)$ in terms of the special polylogarithms (4). For example, we have

$$
\begin{aligned}
& L_{5}(s)= \frac{2}{\sqrt{5}}\left[I_{s}(0.2)-\frac{1}{2^{s}} I_{s}(0.4)+I_{s}(0.6)-\frac{1}{2^{s}} I_{s}(1.2)\right] \\
& L_{-5}(s)= \frac{2}{\sqrt{5}}\left(1+\frac{1}{2^{s}}\right)\left[R_{s}(0.8)-R_{s}(1.6)\right] \\
& L_{10}(s)= \frac{2}{\sqrt{10}}\left[I_{s}(0.1)-\frac{1}{2^{s}} I_{s}(0.2)-I_{s}(0.3)+\frac{1}{2^{s}} I_{s}(0.6)\right. \\
&\left.\quad+I_{s}(0.7)+I_{s}(0.9)-\frac{1}{2^{s}} I_{s}(1.4)-\frac{1}{2^{s}} I_{s}(1.8)\right], \\
& L_{-10}(s)= \frac{2}{\sqrt{10}}\left[R_{s}(0.1)-\frac{1}{2^{s}} R_{s}(0.2)+R_{s}(0.3)-\frac{1}{2^{s}} R_{s}(0.6)\right. \\
&\left.\quad-R_{s}(0.7)+R_{s}(0.9)+\frac{1}{2^{s}} R_{s}(1.4)-\frac{1}{2^{s}} R_{s}(1.8)\right] .
\end{aligned}
$$

The Computation Staff of the Amsterdam Mathematisch Centrum, under the direction of Dr. A. van Wijngaarden, has computed [2] several tables of polylogarithms accurate to $10 \mathrm{D}$. Their Table III gives $R_{s}(\alpha)$ and $I_{s}(\alpha)$ for $s=1(1) 12$ and $\alpha=0(0.01) 2$. The numbers on the right side of $(6)$ for integral $s$ are therefore given explicitly in this table, and thus, with some simple arithmetic, we obtain our Table 1.

TABLE 1

\begin{tabular}{r|c|c|c|c}
\hline$s$ & $L_{5}(s)$ & $L_{-5}(s)$ & $L_{10}(s)$ & $L_{-10}(s)$ \\
\cline { 1 - 1 } 1 & 1.404962946 & 0.6456134114 & 0.9934588266 & 1.150086523 \\
2 & 1.128043325 & 0.8827642541 & 0.9314284985 & 1.092365033 \\
3 & 1.039982136 & 0.9616778624 & 0.9682482537 & 1.034721928 \\
4 & 1.012801468 & 0.9874205162 & 0.9883161275 & 1.012021984 \\
5 & 1.004182100 & 0.9958455012 & 0.9959695576 & 1.004067704 \\
6 & 1.001381310 & 0.9986219811 & 0.9986393802 & 1.001364688 \\
7 & 1.000458601 & 0.9995417817 & 0.9995442414 & 1.000456202 \\
8 & 1.000152606 & 0.9998474373 & 0.9998477867 & 1.000152262 \\
9 & 1.000050832 & 0.9999491729 & 0.9999492226 & 1.000050783 \\
10 & 1.000016939 & 0.9999830616 & 0.9999830687 & 1.000016932 \\
\hline
\end{tabular}


TABLE 2

The Hardy-Littlewood Constants

$$
\begin{aligned}
h_{-10} & =0.67111392 \\
h_{-9} & =0 \\
h_{-8} & =1.85005441 \\
h_{-7} & =0.75737123 \\
h_{-6} & =1.03575587 \\
h_{-5} & =1.77330507 \\
h_{-4} & =0
\end{aligned}
$$

$$
\begin{aligned}
h_{-3} & =1.38342429 \\
h_{-2} & =1.85005441 \\
h_{-1} & =0 \\
h_{0} & =0 \\
h_{1} & =1.37281346 \\
h_{2} & =0.71306310 \\
h_{3} & =1.12073275
\end{aligned}
$$

$$
\begin{aligned}
h_{4} & =1.37281346 \\
h_{5} & =0.52824557 \\
h_{6} & =0.71304162 \\
h_{7} & =1.97304317 \\
h_{8} & =0.71306310 \\
h_{9} & =0.91520897 \\
h_{10} & =1.08240211
\end{aligned}
$$

From Table 1, in turn, we may compute [3], [4] the Hardy-Littlewood constants $h_{a}$ for $a= \pm 5$ and \pm 10 . Together with previously computed values, we may thus complete an 8D table of $h_{a}$ for $a=-10(1) 10$ except for $a= \pm 7$. The $L_{ \pm 7}(s)$, needed to fill this gap, may also be expressed in terms of $I_{s}(\alpha)$ and $R_{s}(\alpha)$, but this time the arguments $\alpha$ are not given explicitly in [2], and elaborate interpolation would be required to obtain comparable precision.

Alternatively, as is known, generalized harmonic series, including $L_{a}(s)$ for integer $s$, may be expressed in terms of the polygamma functions [5], [6]. However, the same difficulty arises for $L_{ \pm 7}(s)$, and again elaborate and laborious interpolation is necessary. At the author's request John W. Wrench, Jr. has kindly computed $L_{7}(2), L_{7}(4), L_{-7}(3)$ and $L_{-7}(5)$ in this way, and these numbers, together with the closed-form $L_{ \pm 7}(s)$, suffice to complete our tabulation of $h_{a}$. This is given in Table 2.

Applied Mathematics Laboratory

David Taylor Model Basin

Washington, D. C. 20007

1. Daniel Shanks \& John W. Wrench, JR., "The calculation of certain Dirichlet series," Math. Comp., v. 17, 1963, p. 136-154; Corrigenda, ibid. p. 488.

2. Staff of the Computation Department, Mathematisch Centrum, Amsterdam, Polylogarithms, Report R24, Part I: Numerical Values, 1954. Reviewed in MTAC, v. 9, 1955, p. 40, UMT 29.

3. Daniel Shanks, "On the conjecture of Hardy \& Littlewood concerning the number of primes of the form $n^{2}+a, "$ Math. Comp., v. 14, 1960, p. 321-332.

4. Daniel Shanks, "Supplementary data and remarks concerning a Hardy-Littlewood conjecture," Math. Comp., v. 17, 1963, p. 188-193.

5. Harold T. Davis, Tables of the Higher Mathematical Functions, vol. 2, Principia Press, Bloomington, Indiana, 1935, p. 14.

6. Eleanor Pairman, "Tables of the digamma and trigamma functions," Tracts for Computers, No. I, Cambridge University Press, 1954.

\section{New Factors of Fermat Numbers}

\section{By Claude P. Wrathall}

Eleven new factors of Fermat numbers $F_{m}=2^{2^{m}}+1$ are listed below. A summary of the present status of the sequence $F_{m}$ is presented in Table 2.

The method used was suggested by Dr. J. L. Selfridge. Simply stated, the method consisted of forming a sieve array to eliminate possible factors divisible by a prime $\leqq 499$. The remaining possible factors were tested to determine if any of the congruence relationships

Received August 22, 1963, revised October 2, 1963. 